\title{
Biochemical screening in newborn siblings of cases of SIDS
}

\author{
Anne Green
}

The association between inherited metabolic disorders and the sudden infant death syndrome (SIDS) received considerable attention in the mid to late 1980s. The earlier reports suggested that as many as $5-10 \%$ of all cases of SIDS in the UK could be due to metabolic disorders. ${ }^{12}$ More recent publications, however, suggest a more conservative estimate that such disorders probably account for fewer than $3 \%$ of all cases of SIDS $^{3-5}$ and thus compare better with the recurrence risk of $2 \%$ quoted in several studies. ${ }^{6}$

The only group of inherited metabolic disorders for which there is good evidence that they are responsible for SIDS are those of fatty acid oxidation. The evidence that other inborn errors can present as SIDS is scanty, ${ }^{78}$ although many defects have the potential to cause unexpected infant death. ${ }^{9}$

Despite the rarity of these disorders it is important to identify those cases where an inherited defect is the likely cause or contributes to the cause of death or a near miss death in infancy. In many cases of so called SIDS there is a history of illness preceeding death and such cases of sudden unexpected death in infancy are equally if not more important to consider.

The finding of fatty change at necropsy ${ }^{1}$ in those infants who have died unexpectedly should stimulate detailed investigation for inherited metabolic disorders. Similarly, paediatricians should consider inherited metabolic disorders in cases of 'near miss' cot death or any patient labelled as having Reye's syndrome and investigate accordingly. ${ }^{10}$ If a specific metabolic disorder is identified, all siblings should then be investigated.

The situation is less clear if a specific disorder is not identified or inherited metabolic disorders are not strongly suspected. Although whole population newborn screening for certain fatty acid oxidation defects is likely to be possible in the future, ${ }^{11}$ it is far from clear at present whether such an approach would satisfy the criteria for population screening and could therefore be justified economically. Currently it is therefore important to consider investigation of higher risk groups (that is siblings)

This discussion specifically considers the investigation of newborn siblings in those families where a previous sibling has died suddenly and unexpectedly, that is it includes both SIDS and sudden unexpected death in infancy.

\section{Biochemical disorders as a possible cause of SIDS}

Inherited metabolic disorders that are known to be associated with acute life threatening events are all possible causes of sudden unexpected death in infancy. ${ }^{9}$ In practice the fatty acid oxidation defects are the only group that have been well documented. ${ }^{12}$

FATTY ACID OXIDATION DEFECTS

Medium chain acyl CoA dehydrogenase (MCAD) deficiency is the most frequently recognised disorder of fatty acid oxidation and probably one of the commonest inborn errors of metabolism. Accurate incidence data is not available, although limited studies on neonatal blood spot specimens suggest that it is commonest in white populations with an incidence possibly as high as one in $10000-15000 .^{1314}$

Presentation of MCAD is usually as hypoketotic hypoglycaemia with a mean age of presentation of 13.5 months. ${ }^{15}$ Patients may be asymptomatic for months or years until a stress related event, for example prolonged fast/viral illness, triggers presentation sometimes as a Reye-like syndrome. There is almost always a history of illness preceeding death.

The diagnosis is unusually based on the finding of characteristic metabolites (medium chain dicarboxylic acids, their metabolites, and respective acyl glycines) in urine by means of gas chromatography - mass spectrometry. ${ }^{16}$ These metabolites may not be present when the infant/ child is well and the diagnosis cannot therefore be unequivocally excluded from urine studies in this situation. Definitive diagnosis requires demonstration of the enzyme deficiency in tissue (liver or cultured skin fibroblasts) or the finding of a specific mutation by molecular studies of DNA extracted from blood or tissue. Assessment of fat oxidation in tissue can be determined by measuring the rate of oxidation of radiolabelled substrates or by more specific electron transfer flavoprotein linked assays. The MCAD gene is located on chromosome 1; a specific point mutation results in substitution of lysine by glutamic acid as a result of replacement of $A$ to $G$ at nucleotide 985. Although other mutations have been found, this $A-G$ substitution is present in a homozygous form in approximately $80-90 \%$ of caes studied." More rarely, long chain fatty acid oxidation defects (long chain acyl 
CoA dehydrogenase and long chain 3-hydroxy acyl CoA dehydrogenase deficiency) have been reported as a cause of unexplained death. ${ }^{17}$ In particular the history of cardiorespiratory arrest in such patients makes these candidate disorders for SIDS. ${ }^{18}$

Finally, disorders of carnitine metabolism or carnitine deficiency resulting in impaired fatty acid oxidation are potential causes of SIDS.

In summary therefore there are a whole range of fatty acid oxidation defects which can present as SIDS. Urinary organic acid analysis using gas chromatography-mass spectrometry will detect most of these cases in symptomatic patients but may miss the diagnosis in a well child. Enzyme assays or DNA analysis are required for definitive diagnosis and are specific for a particular defect or group of disorders.

\section{GLYCOGEN STORAGE DISEASE}

Glycogen storage disease type $I$ is a group of disorders due to deficiency of the hepatic microsomal glucose-6-phosphatase system. ${ }^{19}$ The glucose-6-phosphatase system is a complex multicomponent enzyme system and several different disorders can occur (types Ia, Ib, Ic, and Id), depending on the exact location of the defect. Classical glucose-6-phosphatase deficiency is now termed type Ia glycogen storage disease.

The finding of glycogen storage diseases types $\mathrm{Ia}, \mathrm{Ib}$, and Ic in tissues from patients with SIDS has been reported ${ }^{78}$ and it was suggested that glucose-6-phosphatase deficiency may be a significant cause of SIDS.

The cases reported by Burchell et al, however, were highly selected and more recent data have suggested an alternative explanation for these findings..$^{20}$ Liver glucose-6-phosphatase activity at birth is about $10 \%$ of adult values. In infants born at term the activity rises rapidly after delivery to reach adult values by about 3 days of age. In premature infants this postnatal surge may not occur and glucose-6-phosphatase activity may remain low compared with their normal term counterparts. This apparent failure to switch on glucose-6-phosphatase activity results in impaired mechanisms for maintaining blood glucose homoeostasis. Preliminary results from studies with an animal model suggest that the hormonal and nutritional environment in the perinatal period are critical to the normal development of the glucose-6-phosphatase system. It seems likely therefore that physiological and/or environmental effects on liver glucose-6-phosphatase activity may explain a large percentage of the earlier findings rather than a primary genetic defect.

At present the contribution of the various inherited defects of the glucose-6-phosphatase system as causes of SIDS is unknown. Although there is a suspicion that they may contribute to the causes of SIDS, more research and larger studies are required to produce meaningful incidence data.

OTHERS

Several other inherited metabolic disorders including congenital adrenal hyperplasia, systemic carnitine deficiency, biotinidase deficiency, and maple syrup urine disease can present as sudden, unexpected death. In most cases reported there has usually been a preceeding history of some note. Other disorders including the electron transport chain disorders and disorders of pyruvate metabolism are likely to be included in this group.

A previous history of developmental delay and/or seizures and sudden death after fasting or other catabolic stress such as infection, immunisations, or fever should suggest an inherited defect.

\section{Diagnosis of metabolic disorders \\ INVESTIGATION POSTMORTEM}

The possibility of an inherited metabolic disorder should be considered by the pathologist in all cases of SIDS and especially in those cases of sudden unexpected death in infancy where there has been significant prodromal symptoms. A previous clinical history and or a family history in which more than one infant appears to have died suddenly of unknown cause are among those more likely to have a metabolic cause.

A prompt and careful postmortem examination by a paediatric pathologist with sampling of tissues as quickly as possible after death and appropriate storage at $-70^{\circ} \mathrm{C}$ or below is essential for meaningful biochemical studies. Suitable approaches to sampling for investigation of metabolic disorders have been detailed. ${ }^{921}$

The finding of panlobular fatty change in the liver or fatty change in other tissues (heart, skeletal, muscle) or excess glycogen storage are suggestive of an inherited metabolic disorder and should initiate further metabolic studies. However, the absence of significant fatty infiltration of the liver in a family with a history suggestive of a metabolic disorder should not deter further investigations. ${ }^{22}$

For the majority of cases of SIDS, however, there are no clues from the history or postmortem examination and further metabolic studies are unlikely to be carried out.

HOW TO DIAGNOSE THESE DISORDERS IN ASYMPTOMATIC SIBLINGS

Fatty acid oxidation defects

Fatty acid oxidation defects are detected by the finding of abnormal amounts of dicarboxylic acids and their metabolites in urine. These characteristic findings are present when the child is symptomatic/stressed but may be absent when well. The baby with one of these defects may show abnormalities in the early neonatal period when subject to catabolic stresses. ${ }^{2}$

As part of a national CONI (care of next infant) study into cot deaths, a research project into neonatal screening of siblings by urinary organic acids has been undertaken in Sheffield. Results from the study have shown that screening newborns during the first 72 hours of life for fatty acid oxidation defects is feasible provided that gas chromatography-mass spectrometry is used ( $\mathrm{R} J$ Pollitt, personal communication). Urinary organic acid screening is, however, a 
highly complex investigation and normal babies, without proved defects of fatty acid oxidation, can excrete large quantities of dicarboxylic acids. These findings can be difficult or impossible to differentiate from those found in some types of fatty acid oxidation disorders. The results emphasise the importance of skilled interpretation of urinary organic acids and the difficulties in making a definitive diagnosis based on metabolite profiles. The advantage, however, of the urinary organic acid profile is that it is a nonspecific approach and is capable of detecting a whole range of inborn errors, not only the fatty acid defects.

An alternative approach is to diagnose the fatty acid oxidation defects by specific enzyme or molecular studies. Enzyme assays, however, require tissue (cultured skin fibroblasts/liver/ muscle) and the specialist assays are complex and available in only a handful of centres in the UK. They are not suitable methods for screening. Molecular studies on DNA from blood or tissue will detect specific mutations and currently are applicable only to MCAD. In view of the probable high incidence of MCAD in white populations, and the ability to intervene and influence morbidity and mortality, whole population neonatal screening of MCAD using the neonatal Guthrie blood spot system has been suggested. ${ }^{2}$ This could be achieved by either measurement of the metabolites characteristic of MCAD or DNA analysis. However, before proceeding on a widespread basis, the natural history of the disorder needs to be defined more fully and large scale programmes undertaken to evaluate such an approach.

\section{Glycogen storage disease type I}

Glycogen storage disease type I is characterised by excess glycogen in the liver and diagnosed by measurement of glucose-6-phosphatase activity in liver.

There is no simple 'screening'-type test for this group of disorders, although an increased plasma and urinary lactate concentration may be a useful clue in some cases. It is important to examine at risk siblings for hepatomegaly at 2-3 weeks of age and to measure blood glucose after a four hour fast. Glucagon loading tests can be used in infants and older children.

THE CASE FOR SCREENING IN NEWBOWN SIBLINGS In 1990 there were approximately 1400 cases of SIDS/cot death in the UK (data from the Office of Population Censuses and Surveys). If the case for biochemical screening of all newborn siblings is based on 3\% of cases of SIDS being due to an underlying disorder of fatty acid oxidation it is estimated that there are approximately 40 cases each year of SIDS due to these disorders. Screening in these families with a one in four risk of an affected case for each pregnancy would therefore pick up 10 cases each year assuming that on average each family will have one further child after the sudden infant death; the figure is likely to be less than 10 in practice as not all cases may be detected. Such a policy, although reducing the risk of SIDS recurring in the same family, would therefore have a minimal affect on the overall incidence of SIDS in the UK.

Screening siblings could be based on either urinary or plasma organic acids or molecular studies on DNA. The cost of such screening would be significant. Such investigations require specialist expertise and are available in only a few centres $(<20)$ in the UK. The detailed analysis of urinary organic acids by gas chromatographymass spectrometry is expensive (at least $£ 50$ / specimen) and the workload for an individual laboratory could be substantial, requiring a major investment in resources.

At this moment therefore it would not be justified in the UK to screen all siblings of cases of SIDS, taking into account the likely yield and current technology for screening. The outcome of studies to evaluate neonatal screening for MCAD may influence this approach in the future.

\section{Criteria for investigating newborn siblings}

The case for investigation of siblings should therefore be restricted to:

(1) Siblings who are symptomatic, for example unexplained hypoglycaemia, drowsiness, acidosis, failure to thrive, illness precipitated by infection/stress.

(2) Asymptomatic siblings with one or more of the following:

- Sudden unexpected sibling death with history suggestive of a metabolic disorder, for example progressive lethargy, vomiting, poor oral intake, seizures, hepatomegaly.

- SIDS in a sibling with postmortem findings suggestive of a metabolic disorder, that is widespread fatty change (liver, heart, kidney), glycogen storage.

- More than one case of SIDS in the family.

- Previous sibling with 'Reye-like' illness or unexplained cardiorespiratory arrest.

- Family history: close family member (for example cousin) has had an inherited disorder.

These criteria stress the importance of appropriate and timely postmortem investigation, particularly if there is any pre-existing history suggestive of hypoglycaemia. Currently the extent of investigations can depend on the level of awareness by the pathologist of metabolic disease as a possible cause of SIDS, availability of resources, and availability and logistics of undertaking further investigations.

\section{PRACTICAL ARRANGEMENTS/SPECIMEN} REQUIREMENTS FOR INVESTIGATING NEWBORN SIBLINGS

\section{Symptomatic siblings}

Investigations should be tailored to the particular clinical presentation, ${ }^{23}$ that is to investigate comprehensively for an inherited metabolic disorder.

\section{Asymptomatic siblings}

All siblings who fulfil the criteria above should be investigated, as a minimum for fatty acid oxidation defects. It is suggested that organic acid investigations be undertaken in newborn 
Investigation for inherited metabolic disorders in asymptomatic siblings of cases of SIDS (see criteria)

(1) All cases (see criteria)

(2) History in sibling death Panlobular fatty change

Liver glycogen $^{+++}$

(3) Reye-like illness in sibling
Above plus (if above investigations negative): - Skin biopsy (fat oxidation studies)

- Consider fast when older (under close supervision) Above plus:

- Examine for hepatomegaly

- Preprandial blood glucose and lactate (4 hour fast) Consider:

- Glucagon tolerance test (depending on age)

- Liver glucose-6-phosphatase

Above plus:

- Urinary and plasma amino acids

- Urinary orotic acid

- Consider skin biopsy (fat oxidation studies)

- Consider fast when older (under close supervision)

*Although urinary organic acids and their metabolites are the preferred method of investigation for these disorders in the UK, in other countries measurement of plasma organic acids may be performed as well.

$\mathrm{GC} / \mathrm{MS}=$ gas chromatography-mass spectrometry

$$
\begin{aligned}
& \text { ^Urinary organic acids (GC/MS) } \\
& \text { - MCAD metabolites } \\
& \text { Blood } \\
& \text { - DNA (MCAD mutation) } \\
& \text { - Carnitine }
\end{aligned}
$$

defect could enable prenatal diagnosis or institution of treatment in an asymptomatic sibling to prevent hypoglycaemia and reduce mortality/ morbidity.

All cases of SIDS should be properly investigated postmortem to look for evidence of metabolic disorders and a careful history taken to elicit evidence of pre-existing problems. Although it is currently not appropriate to screen all siblings of cases of SIDS for metabolic disorders, certain high risk groups should be selected for detailed biochemical investigation. The situation may change in the future when results of studies to assess the true incidence and natural history of some of these inherited metabolic disorders have been completed.

*Holton JB, Pennock CA, Walter JA. UK directory of laboratories diagnosing inborn errors of metabolism. 4th Ed. 1991. Obtainable from Dr Holton, from Dr Holton, Biochemistry Department,

siblings in urine collected within the first 72 hours of life. If this time window is missed, an early morning urine should be collected. If the results of the urinary organic acid investigations are negative, blood should be taken for DNA studies (MCAD) and plasma free and acyl carnitine.

Further investigations may be indicated, depending on the particular circumstances of the case (table). If there is a very strong suspicion of a fat oxidation defect, then one should consider undertaking a diagnostic fast (under close medical supervision) with measurement of organic acids, acyl carnitines, and intermediatry metabolites. Extensive investigations are recommended in siblings where the SIDS case has been shown to have widespread fatty change in tissues.

\section{Specimen requirements}

A fresh random urine (no preservatives) is required (ideally $5 \mathrm{ml}$ ). Specimens should be stored deep frozen as soon as possible after collection and transported to the laboratory deep frozen. The request should be sent to a specialist laboratory with expertise in gas chromatographymass spectrometry*. The request should be made specific - MCAD deficiency - and full details of SIDS history should be provided. A 'standard' urinary organic acid investigation may not include gas chromatography-mass spectrometry analysis for the specific metabolites found in MCAD and therefore may miss the diagnosis.

Blood ( $5 \mathrm{ml}$ collected in EDTA) for carnitine and DNA (some laboratories may be able to perform this investigation on a Guthrie blood spot) should be sent to a specialist laboratory undertaking these tests; the laboratory performing organic acid measurements should be able to advise on where these tests are available.

\section{Conclusion}

Inherited metabolic disorders are a rare but important cause of SIDS. For those families at risk, the implications for further pregnancies are enormous. Diagnosis of a fatty acid oxidation
1 Howat AJ, Bennett MJ, Variend S, Shaw L, Engel PC. Defects of metabolism of fatty acids in the sudden infan death syndrome. $B M \mathcal{F}$ 1985; $290: 1771-3$.

2 Bennett MJ, Variend S, Pollit RJ. Screening siblings for inborn errors of fatty acid metabolism in families with inborn errors of fatty acid metabolism in families

3 Divry P, Vianey-Liaud C, Jakobs C, Ten-Brink HJ, Dutruge J, Gilly R. Sudden infant death syndrome: organic acid profiles in cerebrospinal fluid from 47 children and the occurrence of $\mathrm{N}$-acetyl aspartic acid. $\mathcal{F}$ Inherited Metab Dis 1990; 13: 3303-32.

4 Holton JB, Allan JT, Green CA, et al. Inherited metabolic diseases in the sudden infant death syndrome. Arch Dis Child 1991; 66: 1315-7.

5 Bonham JR, Downing $M$. Metabolic deficiency and SIDS f Clin Pathol 1992; 45 (suppl): 33-8.

6 Beal SM, Blundell HL. Recurrence incidence of sudden infant death syndrome. Arch Dis Child 1988; 63: 924-30.

7 Harpey JP, Charpentier C, Paturneau-Jouas M. Sudden infant death syndrome and inherited disorders of fat metabolism. Lancet 1986; ii: 1332 .

8 Burchell A, Bell JE, Busuttil A, Hume R. Hepatic microsomal glucose-6-phosphatase system and sudden infant death syndrome. Lancet 1989; ii: 291-4.

9 Emery JL, Howat AJ, Ariend S, Vawter GF. Investigation of inborn errors of metabolism in unexpected infant deaths. Lancet 1988; ii: 29-31.

10 Green A, Hall SM. Investigation of metabolic disorders syndrome. Arch Dis Child 1992; 67: 1313-7.

11 Yokoto I, Coates PM, Hale DE, Rinaldo P, Tanaka K Molecular survey of a prevalent mutation, 985 A-to-G transition, and identification of five infrequent mutations in transition, and identications in the medium-chain acyl CoA dehydrogenase (MCAD) gen 1991; 49: 1280-91.

12 Anonymous. Sudden infant deaths and inherited disorders of fat oxidation [Editorial]. Lancet 1986; ii: 1073-5.

13 Matsubara Y, Narisawa K, Tada K, et al. Prevalence of K329E mutation in medium-chain acyl-CoA-dehydrogenase gen determined from Guthrie cards. Lancet 1991; 338: 552-3.

14 Blakemore AIF, Singleton H, Pollitt RJ, et al. Frequency of the G985 MCAD mutation in the general population. Lance 1991; 337: 298-9.

15 Touma EH, Charpentier C. Medium chain acyl CoA dehydrogenase deficiency. Arch Dis Child 1992; 67: 142-5.

16 Bennett MJ. The laboratory diagnosis of inborn errors of mitochondrial fatty acid oxidation. Ann Clin Biochem 1990; 27: 519-31.

17 Wanders RJA, Duran M, Ijlst L, et al. Sudden infant death and long chain 3-hydroxy acyl CoA dehydrogenase. Lancet and long; ii: $52-3$.

18 Hale DE, Batshaw ML, Coates PM, et al. Long-chain acyl CoA dehydrogenase deficiency: an inherited cause of nonCoA dehydrogenase deficiency: an inherited cause of

19 Burchell A, Waddel ID. Genetic deficiencies of the hepatic microsomal glucose 6 phosphatase system. In: Randel PJ, microsomal glucose 6 phosphatase system. In: Randel PJ

20 Burchell A, Lyall, Busuttil A, Bell JE, Hume R. Glucose metabolism and hypoglycaemia in SIDS. F Clin Pathol 1992

21 Norman MG, Taylor GP, Clarke LA. Sudden, unexpected, natural death in childhood. Pediatr Pathol 1990; 10: 769-84.

22 Losty HC, Lee P, Alfaham M, Gray OP, Leonard JV. Fatty infiltration in the liver in medium chain acyl CoA dehydrogenase deficiency. Arch Dis Child 1991; 66: 727-8.

23 Saudubray JM, Ogier $\mathbf{H}$. Clinical approach to inherited metabolic disorders. In: Fernandes J, Saudubray JM, Tada K. Inborn metabolic diseases. Berlin: Springer-Verlag, 1990 3-25. John Libbey, 1990: 93-110. 45 (suppl): $39-45$. 